\title{
ON RELATIVE HOMOLOGICAL ALGEBRA OF FROBENIUS EXTENSIONS
}

\author{
KAZUHIKO HIRATA
}

In this paper we study two features of relative homological algebra of Frobenius extensions, the one is the relative dimension and the other is the relative complete resolution. The theory of Frobenius extensions was developed by F. Kasch [4] as a generalization of Frobenius algebras. We deal with only Frobenius extensions of finite rank (see $\S 2$ for the definition).

Let $P$ be a Frobenius extension ring of its subring $Q$. In $\S 3$, we prove that the relative global dimension of $(P, Q)$ is 0 or $\infty$, and further $P$ is an algebra over a commutative ring, the relative dimension of $(P, Q)$ is 0 or $x$.

For a Frobenius algebra $A$ over a field $K, \mathrm{~T}$. Nakayama [5] has constructed a complete resolution for $A$ as a generalization of that for finite groups. On the other hand, a relative complete resolution for $(G, H)$, where $G$ is a group and $H$ is a subgroup of finite index, has been constructed by $G$, Hochschild [3], and in this case the group ring $Z(G)$ is a Frobenius extension algebra of $Z(H)$. In general the ordinary homology and cohomology of a supplemented $K$-algebra ( $K$ is a commutative ring) is obtained from the Hochschild homology and cohomology groups, if the algebra is $K$-projective. We show that this holds without any assumption in the relative case $(\$ 4)$. In particular, the relative homology of group extensions can be treated in the form of the relative homology of algebras. Now, we construct a relative complete resolution for Frobenius extension algebras $(\S 5)$, as the unification of that for Frobenius algebras and that for group extensions.

Throughout this paper we assume that all rings have a unit element which operates as the identity on all modules, and subrings contain the unit element.

The author wishes to express his hearty thanks to A. Hattori for his kind leading.

Received February 5, 1959. 


\section{§1. Duality}

This section is a generalization of $\S 1$ of [2] to the case where the ground ring is not commutative. Let $S$ be a ring. For a right $S$-module $A$, we define the dual module

$$
A^{\circ}=\operatorname{Hom}_{s}(A, S) \text {. }
$$

This is a left $S$-module by defining

$$
(x \cdot \alpha)(a)=x \alpha(a)
$$

for $x \in S, \alpha \in A^{\circ}$ and $a \in A$.

For a left $S$-module $B$, we define similarly the dual module, which is a right $\mathrm{S}$-module, and we denote it by ${ }^{\circ} \mathrm{B}$.

In the situation $\left(A_{S},{ }_{S} C\right)$, we define

$$
\tau: A \underset{B}{\otimes} C \rightarrow \operatorname{Hom}_{B}\left(A^{\circ}, C\right)
$$

by setting

$$
[\tau(a \otimes c)] \alpha=\alpha(a) c, \quad a \in A, \quad c \in C, \quad \alpha \in A^{\circ} .
$$

$\tau$ is an isomorphism whenever $A$ is $S$-projective and finitely generated. Taking $C=S$, we have

$$
\tau: A \rightarrow^{\circ}\left(A^{\circ}\right)=\operatorname{Hom}_{S}\left(A^{\circ}, S\right)
$$

and $\tau$ is an $S$-isomorphism if $A$ is $S$-projective and finitely generated.

For a right $S$-homomorphism $\varphi: A \rightarrow{ }^{\circ} C$, the transposed left $S$-homomorphism $\varphi^{\prime}: C \rightarrow A^{\circ}$ is defined by setting

$$
\left(\varphi^{\prime}(c)\right) a=(\varphi(a)) c
$$

and $\varphi^{\prime}$ is an isomorphism if $C$ is $S$-projective and finitely generated and if $\varphi$ is an isomorphism.

If the right $S$-module $A$ is at the same time a left $R$-module for other ring $R$, then $A^{\circ}$ is a right $R$-module, $\operatorname{Hom}_{s}\left(A^{\circ}, C\right)$ is a left $R$-module and the homomorphism $\tau$ is a left $R$-homomorphism. Similarly if the left $S$-module $C$ is at the same time a right $R$-module and $\varphi$ is a left $R$-homomorphism, then $\varphi^{\prime}$ is a right $R$-homomorphism.

\section{§2. Frobenius extension rings}

Let $R$ be a ring and $S$ be its subring. Then $R$ is considered as a left or 
a right $S$-module. Suppose $R$ is a left and a right $S$-free module with the same number of finite basis elements. We say that $R$ is a Frobenius extension ring of $S$ when the left and the right regular representations of $R$ in $S$ are equivalent. Equivalently, $R$ is a Frobenius extension of $S$ if there exist a right basis $\left(a_{1}, \ldots, a_{n}\right)$ and a left basis $\left(b_{1}, \ldots, b_{n}\right)$, for which the relation

$$
\left\{\begin{array}{l}
x a_{j}=\sum_{i=1}^{n} a_{i} x_{i j} \\
b_{i} x=\sum_{j=1}^{n} x_{i j} b_{j}
\end{array}\right.
$$

holds for every $x$ in $R$ where $x_{i j} \in S$.

Remark. Our condition to define a Frobenius extension is weaker than the one originally given by Kasch. Our definition which is somewhat formal in character, seems to be natural for our present purpose.

The dual module ${ }^{\circ} R$ is a right $S$-free module with the dual basis $\left\{\beta_{j}\right\}$ of $\left\{b_{i}\right\}$ i.e. $\beta_{j}\left(b_{i}\right)=\delta_{i j}$. It is clear that $R$ is isomorphic to ${ }^{\circ} R$ as a right $S$-module by the map

$$
\varphi: a_{j} \rightarrow \beta_{j} .
$$

We can prove easily that $\varphi$ is a left $R$-isomorphism if and only if $(*)$ holds.

Similarly the dual module $R^{\circ}$ is a left $S$ - and right $R$-module. Let $\left\{\alpha_{i}\right\}$ be the dual basis of $\left\{a_{j}\right\}$. Then the transposed homomorphism $\varphi^{\prime}: R \rightarrow R^{\circ}$ of $\varphi$ is explicitly expressed by

$$
\varphi^{\prime}\left(b_{i}\right)=\alpha_{i},
$$

and $\psi^{\prime}$ is a left $S$ - and right $R$-isomorphism. Thus, we have

Proposition 1. $R$ is a Frobenius extension of $S$ if and only if $R$ is right $S$ - and left $R$-isomorphic to ${ }^{\circ} R$ or left $S$ - and right $R$-isomorphic to $R^{\circ}$.

Let $\varphi(1)=\mu, \varphi^{\prime}(1)=\mu^{\prime}$. Then $\mu=\mu^{\prime}$, and $\mu$ is a two-sided $S$-homomorphism of $R$ in $S$. This is the "Frobenius homomorphism" of $R / S$ (Kasch [4]).

Clearly, for every $x, y$ in $R$, the relation

$$
(\varphi(x)) y=\mu(y x)=\left(\varphi^{\prime}(y)\right) x
$$

holds, and $\left\{a_{j}\right\}$ and $\left\{b_{i}\right\}$ form orthogonal bases with respect to $\mu, \mu\left(b_{i} a_{j}\right)=\delta_{i j}$.

Remark. A Frobenius extension $R$ of $S$ is called symmetric if $\mu(y x)=\mu(x y)$ for every $x, y$ in $R$. But in this case $S$ is necessarily commutative. If $S$ is 
contained in the center of $R$, then it needs not to distinguish between ${ }^{\circ} R$ and $R^{\circ}$, and if further $R / S$ is symmetric, $\varphi=\varphi^{\prime}$.

\section{§. Dimension in Frobenius extension rings}

Proposition 2. If $R$ is a Frobenius extension ring of its subring $S$, we have isomorphisms

$$
\begin{aligned}
& \operatorname{Ext}_{R}^{n}(A, R \underset{s}{\otimes} C) \approx \operatorname{Ext}_{S}^{n}(A, C), \quad(n \geqslant 0), \\
& \operatorname{Ext}_{R}^{n}\left(\operatorname{Hom}_{S}(R, C), A\right) \approx \operatorname{Ext}_{S}^{n}(C, A), \quad(n \geqslant 0),
\end{aligned}
$$

for every left R-module $A$ and every left S-module $C$.

Corollary 3. If $R$ is a Frobenius extension ring of $S$ and $C$ is a left $S$ module, then

$$
\begin{aligned}
& \text { 1. inj. } \operatorname{dim}_{R}(R \underset{S}{\otimes} C) \leqq 1 . \text { inj. } \operatorname{dim}_{S} C, \\
& \text { 1. } \operatorname{dim}_{R}\left(\operatorname{Hom}_{S}(R, C)\right) \leqq 1 . \operatorname{dim}_{S} C .
\end{aligned}
$$

Corollary 4. If $R$ is a Frobenius extension ring of $S$ and $S$ is left selfinjective, then $R$ is left self injective.

Theorem 5. If $R$ is a Frobenius extension ring of $S$ and $A$ is a left $R$ module satisfying $1 . \operatorname{dim}_{R} A<\infty$, then

$$
\text { 1. } \operatorname{dim}_{R} A=1 . \operatorname{dim}_{s} A \text {. }
$$

These are proved by the same way as in [2] and we shall omit the proofs.

A ring is quasi-Frobenius if and only if it is left and right Noetherian and left self-injective (Theorem 18, [2]). In the present case, since $R$ is left and right finitely $S$-generated, we have by Corollary 4

Theorem 6. If $R$ is a Frobenius extension of $S$ and $S$ is a quasi-Frobenius ring, then $R$ is a quasi-Frobenius ring (Satz 10, [1]).

G. Hochschild [3] has introduced the notion of relative dimension for modules, algebras etc., which we shall now consider in the case of Frobenius extensions. We shall write 'gl.dim' and 'dim' instead of $d($,$) and c($,$) of$ Hochschild.

Profosition 7. If $R$ is a Frobenius extension of $S$, then

$$
\begin{aligned}
\operatorname{Ext}_{(R, S)}^{n}\left(A, R \otimes_{S} C\right) & =0 & & (n>0), \\
& =\operatorname{Hom}_{s}(A, C) & & (n=0),
\end{aligned}
$$




$$
\begin{aligned}
\operatorname{Ext}_{(R, s)}^{n}\left(\operatorname{Hom}_{S}(R, C), A\right) & =0 & & (n>0), \\
& =\operatorname{Hom}_{S}(C, A) & & (n=0),
\end{aligned}
$$

for every left $R$-module $A$ and every left S-module $C$.

Proof. Using the isomorphism

$$
\begin{aligned}
\operatorname{Hom}_{R}(A, R \underset{S}{\otimes} C) & \approx \operatorname{Hom}_{R}\left(A, \operatorname{Hom}_{S}\left(R^{\circ}, C\right)\right) \approx \operatorname{Hom}_{R}\left(A, \operatorname{Hom}_{s}(R, C)\right) \\
& \approx \operatorname{Hom}_{S}\left(R \otimes \mathbb{R}_{A} A, C\right) \approx \operatorname{Hom}_{S}(A, C)
\end{aligned}
$$

we have the conclusion of first part for $n=0$. Now replace $A$ by an $(R, S)$ projective resolution $X$ of $A$ and passing to homology we have

$$
\operatorname{Ext}_{(R, S)}^{n}(A, R \underset{S}{\otimes} C) \approx H^{n}\left(\operatorname{Hom}_{S}(X, C)\right) .
$$

Since $X$ is $S$-split, $H^{n}\left(\operatorname{Hom}_{S}(X, C)\right)=0$ for $n>0$, we have proved the first part. Using the isomorphism

$$
\begin{aligned}
\operatorname{Hom}_{R}\left(\operatorname{Hom}_{s}(R, C), A\right) & \approx \operatorname{Hom}_{R}\left(\operatorname{Hom}_{S}\left(R^{\circ}, C\right), A\right) \approx \operatorname{Hom}_{R}(R \otimes C, A) \\
& \approx \operatorname{Hom}_{S}\left(C, \operatorname{Hom}_{R}(R, A)\right) \approx \operatorname{Hom}_{S}(C, A)
\end{aligned}
$$

we can prove the second part of Proposition 7 .

Corollary 8. If $R$ is a Frobenius extension ring of $S$ and $C$ is a left $S$. module, then

$$
\begin{aligned}
& \text { 1. inj. } \operatorname{dim}_{(R, S)}(R \otimes C)=0, \\
& \text { 1. } \operatorname{dim}_{(R, S)}\left(\operatorname{Hom}_{S}(R, C)\right)=0 .
\end{aligned}
$$

Remark. These are immediate consequences of Proposition 19 below.

Lemma 9. Let $R$ be a Frobenius extension ring of $S$ and $A$ be a left $R$ module. If $1 \cdot \operatorname{dim}_{(R, S)} A<\infty$, then $1 . \operatorname{dim}_{(R, S)} A=0 . \quad$ Similarly if 1. inj. $\operatorname{dim}_{(R, S)} A$ $<\infty$, then 1. inj. $\operatorname{dim}_{(R, S)} A=0$.

Proof. Let $1 . \operatorname{dim}_{(R, s)} A=n<\infty$ and $C$ be any left $R$-module. From the standard $(R, S)$-exact sequence

$$
0 \rightarrow B \rightarrow R \underset{S}{\otimes C} \rightarrow C \rightarrow 0
$$

we have an exact sequence

$$
\cdots \rightarrow \operatorname{Ext}_{(R, S)}^{n}(A, B) \rightarrow \operatorname{Ext}_{(R, S)}^{n}(A, R \underset{S}{\otimes} C) \rightarrow \operatorname{Ext}_{(R, S)}^{n}(A, C) \rightarrow 0 .
$$

By Corollary 8, $\operatorname{Ext}_{(R, S)}^{n}(A, R \underset{S}{\otimes} C)=0$ whence $\operatorname{Ext}_{(R, S)}^{n}(A, C)=0$ for $n>0$. 
The second part can be proved in a similar way.

Above considerations are also valid for right $R$-modules and right $S$-modules by suitable revisions.

By Lemma 9, we have

THEOREM 10. If $R$ is a Frobenius extension ring of $S$, then

$$
\begin{aligned}
& \text { 1. } g l \cdot \operatorname{dim}(R, S)=0 \text { or } \infty, \\
& \text { r. } \operatorname{gl} \cdot \operatorname{dim}(R, S)=0 \text { or } \infty .
\end{aligned}
$$

Next we consider Frobenius extension algebras. Let $P$ be a $K$-algebra and $Q$ be its subalgebra, where $K$ is a commutative ring. We say that $P$ is a Frobenius extension algebra of $Q$ if $P$ is a Frobenius extension of $Q$ as a ring. Let $R=P \underset{K}{\otimes} P^{\prime}$, the enveloping algebra of $P$, and $S=Q \underset{K}{\otimes} P^{\prime}$, where $P^{\prime}$ is anti-isomorphic to $P$ by the map $x \rightarrow x^{\prime}, x \in P$.

Proposition 11. If $P$ is a Frobenius extension algebra of $Q$, then $R$ is so over $S$.

Proof. Let

$$
P=\sum_{j=1}^{n} a_{j} Q=\sum_{i=1}^{n} Q b_{i}
$$

and suppose that $\left\{a_{j}\right\}$ and $\left\{b_{i}\right\}$ form orthogonal bases. Then

$$
R=\sum_{j=1}^{n}\left(a_{j} \otimes 1^{\prime}\right)\left(Q \underset{K}{\otimes} P^{\prime}\right)=\sum_{i=1}^{n}\left(Q \underset{K^{\prime}}{\otimes} P^{\prime}\right)\left(b_{i} \otimes 1^{\prime}\right),
$$

$\left\{a_{j} \otimes 1^{\prime}\right\}$ and $\left\{b_{i} \otimes 1^{\prime}\right\}$ form a right and a left $S$-basis of $R$ respectively. Further the left and the right regular representations of $R$ in $S$ with respect to these are the same. So $R$ is a Frobenius extension ring of $S$.

TheOREM 12. If $P$ is a Frobenius extension algebra of $Q$, then

$$
\operatorname{dim}(P, Q)=0 \text { or } \infty \text {. }
$$

Proof. $\operatorname{dim}(P, Q)=1 \cdot \operatorname{dim}_{(R, S)} P=0$ or $\infty$ by Lemma 9.

\section{§4. Relative homology and cohomology of supplemented algebras}

Let $P$ be a supplemented $K$-algebra and $Q$ be its subalgebra. Let $R=P \underset{\kappa}{\otimes} P^{\prime}$ and $S$ be the natural image of $Q \underset{K}{\otimes} P^{\prime}$ in $R$, then a two-sided $P$-module is considered as a left or a right $R$-module. 
Lemma 13. If $A$ is an $(R, S)$-projective left $R$-module, then $A \underset{P}{\otimes} K$ is a left $(P, Q)$-projective module.

Proof. $R \underset{S}{\otimes} A$ is left $R$-isomorphic to $P \underset{Q}{\otimes} A$ by the map $f$ :

$$
f\left(p_{1} \otimes p_{2}^{\prime} \otimes a\right)=p_{1} \otimes a p_{2}, \quad p_{1} \otimes p_{2}^{\prime} \otimes a \in R \otimes_{s} A .
$$

If $A$ is $(R, S)$-projective, then $A$ is isomorphic to an $R$-direct summand of $R \otimes A$ and so of $P \underset{Q}{\otimes} A$. Thus $A \underset{P}{\otimes} K$ is left $P$-isomorphic to a direct summand of $P \underset{Q}{\otimes} A \underset{P}{\otimes} K$. Since $P \underset{Q}{\otimes} A \underset{P}{\otimes} K$ is $(P, Q)$-projective, $A \underset{P}{\otimes} K$ is $(P, Q)$-projective.

Corollary 14. If $X$ is a left $(R, S)$-projective resolution of $P$, then $X \underset{P}{\otimes} K$ is a left $(P, Q)$-projective resolution of $K$.

Proof. Since $X$ is right $P$-split, $X \underset{P}{\otimes} K$ is $(P, Q)$-exact. The $(P, Q)$-projectivity follows from Lemma 13 , and $X \underset{P}{\otimes} K$ is a $(P, Q)$-projective resolution of $K$.

THEOREM 15. For a left P-module $M$ and a right P-module $N$, we have

$$
\begin{aligned}
& H^{n}\left((P, Q), M_{\varepsilon}\right)=\operatorname{Ext}_{(R, s)}^{n}\left(P, M_{\varepsilon}\right) \approx \operatorname{Ext}_{(P, Q)}^{n}(K, M), \\
& \left.H_{n}\left((P, Q),{ }_{\varepsilon} N\right)=\operatorname{Tor}_{n}^{(R, S)}{ }_{\varepsilon} N, P\right) \approx \operatorname{Tor}_{n}^{(P, Q)}(N, K),
\end{aligned}
$$

where $\varepsilon$ is the augmentation belonging to the supplemented $K$-algebra $P$, and $M_{\varepsilon}$ is a two-sided P-module converted into a right P-module by setting $m p=m(\varepsilon p)$, $m \in M, p \in P$, similarly for ${ }_{\varepsilon} N$.

Proof. We have isomorphisms

$$
\begin{aligned}
& \operatorname{Hom}_{R}\left(A, M_{\varepsilon}\right) \approx \operatorname{Hom}_{R}\left(A, \operatorname{Hom}_{K}(K, M)\right) \approx \operatorname{Hom}_{P}(A \underset{P}{\otimes} K, M), \\
& { }_{\varepsilon} N \underset{R}{\otimes} A \approx K \underset{K}{\otimes} N \underset{R}{\otimes} A \approx N \underset{P}{\otimes} A \underset{P}{\otimes} K,
\end{aligned}
$$

for any two-sided $P$-module $A$ (cf. [1], IX, 2.1 and 2.2). Replacing $A$ by a left $(R, S)$-projective resolution $X$ of $P$, and passing to homology we have desired conclusions, by Corollary 14 .

\section{$\S 5$. Relative complete resolution}

PROPOSITION 16. Let $P$ be any K-algebra and $Q$ be its subalgebra. If $A$ is $a(P, Q)$-projective left $P$-module, then $\operatorname{Hom}_{\kappa}(A, C)$ is $a(P, Q)$-injective right $P$-module and $A \underset{K}{\otimes} C$ is a $(P, Q)$-projective left $P$-module for every $K$-module $C$.

Proof. Let 


$$
0 \rightarrow U \rightarrow V
$$

be a $(P, Q)$-exact sequence of right $P$-modules $U$ and $V$. Since this sequence is $Q$-split, it is also $K$-split and we have a $(P, Q)$-exact sequence

$$
\operatorname{Hom}_{K}(V, C) \rightarrow \operatorname{Hom}_{K}(U, C) \rightarrow 0,
$$

of left $P$-modules. Since $A$ is $(P, Q)$-projective

$$
\operatorname{Hom}_{P}\left(A, \operatorname{Hom}_{K}(V, C)\right) \rightarrow \operatorname{Hom}_{P}\left(A, \operatorname{Hom}_{K}(U, C)\right) \rightarrow 0
$$

is exact. But $\operatorname{Hom}_{F}\left(A, \operatorname{Hom}_{K}(V, C)\right)$ and $\operatorname{Hom}_{P}\left(A, \operatorname{Hom}_{K}(U, C)\right)$ are isomorphic to $\operatorname{Hom}_{P}\left(V, \operatorname{Hom}_{K}(A, C)\right)$ and $\operatorname{Hom}_{P}\left(U, \operatorname{Hom}_{K}(A, C)\right)$ respectively by the natural correspondence. So we have an exact sequence

$$
\operatorname{Hom}_{P}\left(V, \operatorname{Hom}_{K}(A, C)\right) \rightarrow \operatorname{Hom}_{P}\left(U, \operatorname{Hom}_{K}(A, C)\right) \rightarrow 0
$$

and $\operatorname{Hom}_{K}(A, C)$ is $(P, Q)$-injective.

The second part can be proved in a similar way.

Lemma 17. Let $P$ be $a$ K-algebra, $Q$ be its subalgebra and $C$ be a left $Q$ and right $P$-module. Then $\operatorname{Hom}_{Q}(P, C)$ (left $Q$-homomorphisms) is $P \underset{K}{\otimes} P^{\prime}$. isomorphic to $\operatorname{Hom}_{Q} \otimes_{K} P^{\prime}\left(P \underset{K}{\otimes} P^{\prime}, C\right)$, and $P \underset{Q}{\otimes} C$ is $P \underset{K}{\otimes} P^{\prime}$-isomorphic to $\left(P \underset{K}{\otimes} P^{\prime}\right) \underset{K}{\otimes} \otimes_{P^{\prime}}$, i.e. $\operatorname{Hom}_{Q}(P, C)$ is $\left(P \underset{K}{\otimes} P^{\prime}, Q \underset{K}{\otimes} P^{\prime}\right)$-injective and $P \underset{Q}{\otimes} C$ is $\left(P \underset{K}{\otimes} P^{\prime}, Q \underset{K}{\otimes} P^{\prime}\right)$-projective.

Proof. Defining $\emptyset: \operatorname{Hom}_{Q}(P, C) \rightarrow \operatorname{Hom}_{Q} \underset{K}{\otimes P^{\prime}}\left(P \underset{K}{\otimes} P^{\prime}, C\right)$ and $\Psi:$ $\operatorname{Hom}_{Q} \underset{K}{\otimes} P^{\prime}\left(P \underset{K}{\otimes} P^{\prime}, C\right) \rightarrow \operatorname{Hom}_{Q}(P, C)$ by setting

$$
\begin{array}{ll}
(\emptyset f)\left(a \otimes b^{\prime}\right)=f(a) b, & f \in \operatorname{Hom}_{Q}(P, C), \quad a \otimes b^{\prime} \in P \underset{K}{\otimes} P^{\prime}, \\
(\Psi g)(a)=g\left(a \otimes 1^{\prime}\right), & g \in \operatorname{Hom}_{Q} \underset{K}{\otimes}\left(P \otimes_{K} P^{\prime}, C\right), \quad a \in P,
\end{array}
$$

we have the first part of Lemma 17 . The second part is similar.

Lemma 18. With the same $P$ and $Q$ as in Lemma 17, $\operatorname{Hom}_{P}(A \underset{Q}{\otimes} P, P)$ is $P \underset{K}{\otimes} P^{\prime}$-isomorphic to $\operatorname{Hom}_{Q}\left(P, \operatorname{Hom}_{P}(A, P)\right)$, where $A$ is a left $P$-and right Q-module.

Proof. The correspondence is the same as in [1], II, 5.2.

Proposition 19. If $R$ is a Frobenius extension ring of $S$ and $A$ is a left $R$-module, then the following conditions are equivalent:

(i) There is a homomorphism $h$ in $\operatorname{Hom}_{S}(A, A)$ such that $\sum_{i=1}^{n} a_{i} h b_{i}=1_{A}$, 
the identity map of $A$, where $\left\{a_{i}\right\}$ and $\left\{b_{i}\right\}$ are orthogonal S-bases of $R$.

(ii) $A$ is ( $R, S)$-projective.

(iii) $A$ is $(R, S)$-injective.

This is Satz 12, in [4], and we shall omit the proof.

For a right $R$-module $A$, the condition ( $i$ ) is interchanged to $\sum_{i=1}^{n} b_{i} h a_{i}=1_{A}$.

The Frobenius homomorphism $\mu$ of $R$ in $S$, so in $R$, satisfies the condition (i), so that we have

TheOREm 20. If $R$ is a Frobenius extension ring of $S$, then

$$
\begin{aligned}
& \text { 1. } \operatorname{dim}_{(R, s)} R=\text { r. } \operatorname{dim}_{(R, s)} R=0, \\
& \text { 1. inj. } \operatorname{dim}_{(R, s)} R=\text { r. inj. } \operatorname{dim}_{(R, s)} R=0 .
\end{aligned}
$$

Under these preparations we now construct the relative complete resolution for Frobenius extension $K$-algebra $P$ of its subalgebra $Q$. We set $R=P \underset{K}{\otimes} P^{\prime}$ and $S=Q \underset{K}{\otimes} P^{\prime}$.

Let $X_{n}$ be the $(n+2)$-fold tensor product of $P$ over $Q$ for $n \geqslant 0 . \quad X_{n}$ has a two-sided $P$-module structure by setting

$$
\begin{aligned}
& p\left(x_{0} \otimes \cdots \otimes x_{n+1}\right)=p x_{0} \otimes \cdots \otimes x_{n+1}, \\
& \left(x_{0} \otimes \cdots \otimes x_{n+1}\right) p=x_{0} \otimes \cdots \otimes x_{n+1} p,
\end{aligned}
$$

for $p \in P, x_{0} \otimes \cdots \otimes x_{n+1} \in X_{n}$. The differentiations $u_{n}: X_{n} \rightarrow X_{n-1}(n>0)$, and $\bar{u}_{0}: X_{0} \rightarrow P$ are defined by setting

$$
\begin{aligned}
& u_{n}\left(x_{0} \otimes \cdots \otimes x_{n+1}\right)=\sum_{i=0}^{n}(-1)^{i} x_{0} \otimes \cdots \otimes x_{i} x_{i+1} \otimes \cdots \otimes x_{n+1}, \\
& \bar{u}_{0}\left(x_{0} \otimes x_{1}\right)=x_{0} x_{1} .
\end{aligned}
$$

If we define $v_{n}: X_{n} \rightarrow X_{n+1}(n \geq 0)$, and $\ddot{v}_{-1}: P \rightarrow X_{0}$ by setting

$$
\begin{aligned}
& v_{n}\left(x_{0} \otimes \cdots \otimes x_{n+1}\right)=1 \otimes x_{0} \otimes \cdots \otimes x_{n+1}, \\
& \bar{v}_{-1}(x)=1 \otimes x,
\end{aligned}
$$

then we have

$$
u_{n+1} v_{n}+v_{n-1} u_{n}=1, \quad(n>0) ; \quad u_{1} v_{0}+\bar{v}_{-1} \bar{u}_{0}=1 .
$$

Further $X_{n}$ is $R$-isomorphic to $R \otimes X_{n+1}(n>0)$, and $X_{0}$ is $R$-isomorphic to $R \otimes_{S} P$. So that $X^{+}=\sum_{n \geqq 0} X_{n}$ is a left $(R, S)$-projective resolution of $P$ (see [3]).

On the other hand, if we define $v_{n}^{\prime}: X_{n} \rightarrow X_{n+1}(n \geq 0)$ and $v_{-1}^{\prime}: P \rightarrow X_{0}$ by setting 


$$
\begin{aligned}
& v_{n}^{\prime}\left(x_{0} \otimes \cdots \otimes x_{n+1}\right)=(-1)^{n-1} x_{0} \otimes \cdots \otimes x_{n+1} \otimes 1, \\
& v_{-1}^{\prime}(x)=x \otimes 1
\end{aligned}
$$

then we have

$$
u_{n+1} v_{n}^{\prime}+v_{n-1}^{\prime} u_{n}=1, \quad(n>0) ; \quad u_{1} v_{0}^{\prime}+v_{-1}^{\prime} u_{0}=1,
$$

and $X^{+}$is a right $(R, S)$-exact sequence.

Now we define the negative complex $X_{-n}(n \geqslant 1)$ by setting

$$
X_{-n}=\operatorname{Hom}_{P}\left(X_{n-1}, P\right),
$$

where homomorphisms are left $P$-homomorphisms, and $X_{-n}$ is a two-sided $P$ module by the usual way. Since the sequence $X^{+}$is right $(R, S)$-exact, it is left $P$-split and we can prove that $X^{-}=\sum_{n \Xi 1} X_{-n}$ is a left $(R, S)$-exact sequence. By Lemma 18, $\operatorname{Hom}_{P}\left(P \underset{Q}{\otimes} \cdots \otimes_{Q} P, P\right)$ is $R$-isomorphic to $\operatorname{Hom}_{Q}\left(P, \operatorname{Hom}_{P}(P \underset{Q}{\otimes}\right.$ $\cdots \otimes P, P))$; and the latter is left $(R, S)$-injective by Lemma 17. Further it is $(R, S)$-projective by Proposition 19, since $R$ is a Frobenius extension ring of $S$ by Proposition 11. By the map \& : $P \rightarrow \operatorname{Hom}_{P}(P, P)$

$$
(p)(x)=x p, \quad x, p \in P,
$$

$P$ is two-sided isomorphic to $\operatorname{Hom}_{P}(P, P)$. We define $u_{0}: X_{\theta} \rightarrow X_{-1}$ as the composition of

$$
X_{0} \stackrel{\bar{u}_{0}}{\longrightarrow} P \stackrel{\iota}{\longrightarrow} \operatorname{Hom}_{P}(P, P) \stackrel{\bar{u}_{0}^{*}}{\longrightarrow} \operatorname{Hom}_{P}\left(X_{0}, P\right)=X_{-1},
$$

where $\bar{u}_{0}^{*}$ is the homomorphism induced from $\bar{u}_{0}$. S-homomorphism $v_{-1}: X_{-1} \rightarrow X_{0}$. can also be defined as the composition $\bar{v}_{-1} \iota^{-1} v_{-1}^{\prime *}$. Thus we have a relative complete resolution for $P$.

Now we compare with the case of ordinary Frobenius algebra over a field $K$. In this case the negative part are given by $\operatorname{Hom}_{K}\left(P \underset{K}{\otimes} \cdots \otimes_{K} P, K\right)$ modifying the right $P$-module structure by the Nakayama's automorphism * belonging to $P$ (see $[5]$ ).

We have an isomorphism

$$
\operatorname{Hom}_{K}(P \underset{K}{\otimes}(P \underset{K}{\otimes} \cdots \underset{K}{\otimes} P), K) \approx \operatorname{Hom}_{K}\left(P \underset{K}{\otimes} \cdots \underset{K}{\otimes} P, \operatorname{Hom}_{K}(P, K)\right) .
$$

and the right $P$-module structure of right hand side is defined from that of $\operatorname{Hom}_{K}(P, K)$.

On the other hand, in our case we have an isomorphism 


$$
\begin{aligned}
\operatorname{Hom}_{P}\left(P \underset{K}{\otimes}\left(P \underset{K}{\otimes} \cdots \otimes_{K}^{\otimes} P\right), P\right) & \approx \operatorname{Hom}_{K}\left(P \underset{K}{\otimes} \cdots \otimes_{K} P, \operatorname{Hom}_{K}(P, P)\right) \\
& \approx \operatorname{Hom}_{K}\left(P \underset{K}{\otimes} \cdots \otimes_{K} P, P\right)
\end{aligned}
$$

and the right $P$-module structure of last term is defined from that of $P$. But $P$ is right $P$-isomorphic to $\operatorname{Hom}_{K}(P, K)$ when $\operatorname{Hom}_{K}(P, K)$ is modified by *. So the modified $\operatorname{Hom}_{K}\left(P \underset{K}{\otimes} \cdots \otimes_{K} P, K\right)$ is isomorphic to $\operatorname{Hom}_{P}\left(P \underset{K}{\otimes} \cdots \otimes_{\Lambda^{\prime}} P, P\right)$.

Next we consider the case that $P$ is a supplemented $K$-algebra with an antipodism. Let $X^{+}$be the same as above. Then $X^{+} \underset{P}{\otimes}$ is a left $(P, Q)$ projective resolution of $K$ by Corollary 14. By proposition 16, $\operatorname{Hom}_{K}\left(X^{+} \otimes_{P} K, K\right)$ is a $(P, Q)$-injective right $P$-module. Since $X^{+} \underset{P}{\otimes} K$ is $Q$-split, it is $K$-split and we can prove that $\operatorname{Hom}_{K}\left(X^{+} \underset{P}{\otimes} K, K\right)$ is $(P, Q)$-exact. By Proposition 19 and by the antipodism, $\operatorname{Hom}_{K}\left(X^{+} \underset{P}{\otimes} K, K\right)$ is a $(P, Q)$-projective left $P$-module. Thus we can construct a relative complete resolution for $(P, Q)$ of $K$, using $\operatorname{Hom}_{K}\left(X^{+} \underset{P}{\otimes} K, K\right)$ as the negative dimension part.

We shall show that $\operatorname{Hom}_{P}(P \underset{Q}{\otimes} \cdots \otimes \underset{Q}{\otimes} P, P) \underset{P}{\otimes} K$ is left $P$-isomorphic to $\operatorname{Hom}_{K}\left(P \underset{Q}{\otimes} \cdots \otimes \otimes_{Q} P \underset{P}{\otimes} K, K\right)$ in the case $P=K(G)$ and $Q=K(H)$ where $G$ is a group, $H$ is a subgroup of finite index. Let $G=\bigcup_{i=1}^{r} g_{i} H=\bigcup_{i=1}^{r} H g_{i}^{-1}$ be decompositions to right and left cosets. We fix the $g_{i}$ 's once for all. Then the $(n+2)$ fold tensor product $P \underset{Q}{\otimes} \cdots \otimes_{Q} P$ is a left free $P$-module with the basis $\left\{1 \otimes g_{i_{1}}^{-1} \otimes\right.$ $\left.\cdots \otimes g_{i_{n+1}}^{-1} ; i_{k}=1, \ldots, r\right\}$ and $\operatorname{Hom}_{P}\left(P \underset{Q}{\otimes} \cdots \otimes \otimes_{Q} P, P\right) \underset{P}{\otimes} K$ is a free $K$-module with the basis $\left\{\left(1 \otimes g_{i_{1}}^{-1} \otimes \cdots \otimes g_{i_{n+1}}^{-1}\right)^{\wedge} \otimes 1\right\}$, where $\left\{\left(1 \otimes g_{i_{1}}^{-1} \otimes \cdots \otimes g_{i_{n+1}}^{-1}\right)^{\wedge}\right\}$ is the dual basis of $\left\{1 \otimes g_{i_{1}}^{-1} \otimes \cdots \otimes g_{i_{n+1}}^{-1}\right\}$. Similarly $P \otimes \cdots \otimes P$ is a right free $P$-module with the basis $\left\{g_{j_{1}} \otimes \cdots \otimes g g_{n_{2}} \otimes 1 ; j_{k}=1, \ldots, r\right\}$ and $\operatorname{Hom}_{K}(P \underset{Q}{\otimes} \cdots \underset{P}{\otimes} \underset{P}{\otimes} K, K)$ is a free $K$-module with the basis $\left\{\left(g_{j_{1}} \otimes \cdots\right.\right.$ $\left.\left.\otimes g_{j_{n+1}} \otimes 1\right)^{\wedge}\right\} . \quad$ We assign $\quad\left(1 \otimes g_{i_{1}}^{-1} \otimes \cdots \otimes g_{i_{n+1}}^{-1}\right)^{\wedge} \otimes 1 \quad$ to $\quad\left(g_{i_{n+1}} \otimes \cdots\right.$ $\left.\otimes g_{i_{1}} \otimes 1\right)^{\wedge}$. Let $g$ be any element of $G$, then

$$
\begin{aligned}
& \left(g \cdot\left(1 \otimes g_{i_{1}}^{-1} \otimes \cdots \otimes g_{i_{n+1}}^{-1}\right)^{\wedge}\right)\left(1 \otimes g_{j_{1}}^{-1} \otimes \cdots \otimes g_{j_{n+1}}^{-1}\right) \\
& =\left(1 \otimes g_{i_{1}}^{-1} \otimes \cdots \otimes g_{i_{n+1}}^{-1}\right)^{\wedge}\left(1 \otimes g_{j_{1}}^{-1} \otimes \cdots \otimes g_{j_{n+1}}^{-1} g\right) \\
& =\left(1 \otimes g_{i_{1}}^{-1} \otimes \cdots \otimes g_{i_{n+1}}^{-1}\right)^{\wedge}\left(h_{n+1} \otimes g_{k_{1}}^{-1} \otimes \cdots \otimes g_{k_{n+1}}^{-1}\right) \\
& =h_{n+1} \delta_{k_{1} \cdots k_{n+1}}^{i, \cdots i_{n+1}} \text {, }
\end{aligned}
$$

where $h_{n+1}$ is obtained from $g_{j_{n+1}}^{-1} g=h_{1} g_{k_{n+1}}^{-1}, g_{j_{n}}^{-1} h_{1}=h_{2} g_{k_{n}}^{-1}, \ldots, g_{j_{1}}^{-1} h_{n}=h_{n+1} g_{j_{1}}^{-1}$ $\left(h_{i}^{\prime} s \in H\right)$. So we have 


$$
\begin{aligned}
g & \cdot\left(\left(1 \otimes g_{i_{1}}^{-1} \otimes \cdots \otimes g_{i_{n+1}}^{-1}\right) \wedge \otimes 1\right)=g \cdot\left(1 \otimes g_{i_{1}}^{-1} \otimes \cdots \otimes g_{i_{n+1}}^{-1}\right)^{\wedge} \otimes 1 \\
& =\sum_{j_{1} \cdots j_{n+1}}\left(1 \otimes g_{j_{1}}^{-1} \otimes \cdots \otimes g_{j_{n+1}}^{-1}\right) \wedge h_{n+1}^{j_{1} \ldots j_{n+1}} \delta_{k_{1} \cdots k_{n+1}}^{i_{1} \cdots i_{n+1}} \otimes 1 \\
& =\sum_{J_{1} \cdots j_{n+1}}^{j_{1}}\left(1 \otimes g_{j_{1}}^{-1} \otimes \cdots \otimes g_{j_{n+1}}^{-1}\right) \wedge \delta_{k_{1} \cdots k_{n+1}}^{i_{1} \cdots i_{n+1}} \otimes \varepsilon\left(h_{n+1}^{j_{1} \cdots j_{n+1}}\right) .
\end{aligned}
$$

On the other hand

$$
\begin{aligned}
(g & \left.\cdot\left(g_{i_{n_{1}}} \otimes \cdots \otimes g_{i_{1}} \otimes 1\right)^{\wedge}\right)\left(g_{j_{n+1}} \otimes \cdots \otimes g_{j_{1}} \otimes 1\right) \\
\quad & =\left(g_{i_{n+1}} \otimes \cdots \otimes g_{i_{1}} \otimes 1\right)^{\wedge}\left(g^{-1} g_{j_{n+1}} \otimes \cdots \otimes g_{j_{1}} \otimes 1\right) \\
\quad & =\left(g_{i_{n+1}} \otimes \cdots \otimes g_{i_{1}} \otimes 1\right)^{\wedge}\left(g_{k_{n+1}} \otimes \cdots \otimes g_{k_{1}} \otimes \varepsilon\left(h_{n+1}^{-1}\right)\right) .
\end{aligned}
$$

Thus we have

$$
\begin{aligned}
g & \cdot\left(g_{i_{n+1}} \otimes \cdots \otimes g_{i_{1}} \otimes 1\right)^{\wedge} \\
& =\sum_{j_{n+1} \cdots j_{1}}\left(g_{j_{n+1}} \otimes \cdots \otimes g_{j_{1}} \otimes 1\right)^{\wedge} \delta_{k_{n+1} \cdots k_{1}}^{z_{n+1} \cdots i_{1}} \varepsilon\left(h_{n+1}^{-1 j_{n+1} \cdots j_{1}}\right) .
\end{aligned}
$$

Since we may assume that $\varepsilon$ is the unit augmentation, we have a desired isomorphism.

THEOREM 21. For a Frobenius extension algebra of its subalgebra, we can construct a relative complete resolution and this is a generalization of that of Frobenius algebras as well as the relative case of groups.

\section{REFERENCES}

[1] H. Cartan-S. Eilenberg, Homological Algebra, Princeton Univ., 1956.

[2] S. Eilenberg-T. Nakayama, On the Dimension of Modules and Algebras II, Nagoya Math. J. 9 (1955).

[3] G. Hochschild, Relative Homological Algebra, Trans. of Amer. Math. Soc. 82 (1956).

[4] F. Kasch, Grundlagen einer Theorie der Frobeniuserweiterungen, Math. Ann. 127 (1954).

[5] T. Nakayama, On the Complete Cohomology Theory of Frobenius Algebras, Osaka Math. J. 9 (1957).

Tokyo University of Education 